Published in final edited form as:

J Am Acad Orthop Surg. 2013 February ; 21(2): 88-98. doi:10.5435/JAAOS-21-02-88.

\title{
Fracture-dislocations of the Proximal Interphalangeal Joint
}

\author{
John Elfar, MD and Tobias Mann, MD, MSc \\ Department of Orthopaedics and Rehabilitation, University of Rochester, Rochester, NY
}

\begin{abstract}
Fracture-dislocations of the proximal interphalangeal joint encompass a spectrum of injury severity, ranging from injuries that require little intervention to those that require advanced reconstructive surgery for optimal outcome. Three fracture-dislocation patterns are recognized: dorsal, volar, and pilon. Acceptable outcome is dependent on achieving and maintaining a wellaligned and well-reduced joint, re-establishing normal joint kinematics, and restoring motion. Anatomic articular surface reduction is desirable but not absolutely necessary for a good outcome. Treatment depends on both the type of injury and patient-dependent factors. Optimal outcome for a specific injury is predicated on expedient diagnosis and recognition of injury severity, which enables initiation of appropriate management.
\end{abstract}

The proximal interphalangeal joint (PIPJ) is the most commonly injured joint in the hand. ${ }^{1}$ The long lever arm and the joint's exposed position leave it vulnerable to injury. Although most of these injuries are simple ligamentous sprains, more severe fracture-dislocations can occur. Of these, dorsal fracture-dislocations are the most common. PIPJ injuries are often overlooked or dismissed as nothing more than a "jammed" or sprained finger, and treatment may be delayed if the patient "self-treats" the injury. The PIPJ is prone to stiffness following even minor sprains; therefore, early diagnosis and treatment of severe fracture-dislocations is essential to achieve optimal outcomes. ${ }^{2}$

\section{Anatomy}

The bony architecture of the PIPJ allows it to function as a hinge, permitting flexion and extension in the sagittal plane (Figure 1). However, owing to a slight asymmetry between the proximal phalangeal condyles, the middle phalanx supinates with flexion. ${ }^{3}$

The PIPJ has the largest motion arc of the digital joints, normally from $0^{\circ}$ to $120^{\circ}$. Motion at the PIPJ accounts for $85 \%$ of excursion at the fingertip. ${ }^{3}$ Although bony constraints impart some joint stability, the volar plate (VP) and the collateral ligaments (CLs) are the important stabilizers $^{3,4}$ (Figure 2).

The VP is a fibrocartilaginous structure that forms the floor of the PIPJ and is firmly attached to the volar base of the middle phalanx. The central portion of this attachment is thinner than the more substantial lateral portion, which blends with the CLs. ${ }^{4}$ Proximally, the VP forms two swallowtail-like extensions called checkrein ligaments that attach to the proximal phalanx. ${ }^{4}$ The VP is primarily responsible for resisting PIPJ hyperextension; it moves as a unit, gliding proximally with flexion. ${ }^{5}$ This motion has been likened to the gliding of a plate of armor as opposed to the accordion-like collapse of the VP at the 
metacarpophalangeal (MCP) joint. ${ }^{6}$ The CLs are the primary restraints to radial- and ulnardirected forces and are composed of the proper and accessory collateral ligaments ${ }^{3,5}$ (Figure $3)$.

\section{Mechanism of Injury}

PIPJ fracture-dislocations are caused by two basic mechanisms: avulsion or impaction shear.

${ }^{7}$ The direction of dislocation is used to categorize the injury as a dorsal fracture-dislocation (ie, dorsal dislocation with fracture of the middle phalangeal volar lip) or a volar fracturedislocation (ie, volar dislocation with fracture of the dorsal lip of the middle phalanx). The third category is a pilon injury in which both the volar and dorsal lips are involved in the fracture. ${ }^{8}$

\section{Dorsal Fracture-dislocation}

Extension of the PIPJ places tension on the VP. In hyperextension, the VP fails either by rupture or avulsion of the middle phalangeal volar lip. Avulsion fractures vary in size and displacement, but typically there is no comminution. ${ }^{9}$ Alternatively, an axial load applied to the digit with the PIPJ in flexion drives the middle phalanx into the head of the proximal phalanx. Because of the PIPJ flexion, the axial load creates a shearing force across the middle phalangeal base, resulting in a fracture of the volar lip. ${ }^{10}$ As the fractured base is driven into the head of the proximal phalanx, the metaphyseal bone of the middle phalanx is impacted and often comminuted. ${ }^{7,9,10}$ Regardless of mechanism, the restraint afforded by the VP is lost and, unless the fracture fragment is small, the buttressing effect of the volar margin of the middle phalanx is also lost (Figure 4, A). When these static restraints are lost, the forces acting on the PIPJ will displace the middle phalanx dorsally (Figure 5).

\section{Volar Fracture-dislocation}

Volar fracture-dislocations are the result of a mechanism analogous to but opposite that of dorsal fracture-dislocations. Hyperflexion of the PIPJ stresses the central slip, which fails due to rupture or avulsion of the dorsal lip of the middle phalanx. ${ }^{9}$ Impaction-shear injuries are caused by axial loading of the finger with the PIPJ in extension. ${ }^{9,10}$ As with dorsal fracture-dislocations, the articular surface can be both impacted and comminuted. When enough of the dorsal lip is disrupted, the bony constraints are lost (Figure 4, B). Owing to the unopposed action of the flexor digitorum superficialis, the middle phalanx is displaced volarly. ${ }^{11}$

\section{Pilon Injuries}

When a sufficient amount of energy is imparted to the middle phalangeal base via an axial load on the extended PIPJ, a pilon injury may result (Figure 4, C). Stern et $\mathrm{al}^{8}$ defined these injuries as fractures involving both the volar and dorsal cortical margins of the middle phalangeal base, with extensive comminution and depression of the central articular surface. 


\section{Classification}

Fracture-dislocations of the PIPJ are classified based on the direction of dislocation of the middle phalanx and their stability following reduction. The amount of articular surface involvement can often be used to predict stability ${ }^{11,12}$ (Figure 6). Clinically, stability of PIPJ fracture-dislocations is defined by the ability to maintain a well-aligned reduction. Dorsal fracture-dislocations have increased stability with increasing flexion. When $>30^{\circ}$ of flexion is required to maintain reduction, the injury is considered unstable. ${ }^{7}$ Table 1 outlines a classification scheme for fracture-dislocations of the PIPJ.

Volar fracture-dislocations are classified in a similar manner. The ability to maintain a reduced joint in extension defines whether the injury is stable. ${ }^{9}$ In the case of nondisplaced or minimally displaced avulsion fractures, the joint may be stable with up to $50 \%$ of dorsal articular surface involvement. ${ }^{9}$ These injuries have the greatest stability in extension. Pilon fractures involve the entire articular surface and, in the setting of fracture displacement, these injuries are unstable. ${ }^{8}$

\section{Clinical Evaluation}

Evaluation begins with a thorough, focused history and physical examination. Salient features include the time from injury to presentation, presence and nature of any joint instability, mechanism of injury, and history of trauma to the digit.

\section{Imaging}

Appropriate imaging includes standard AP and lateral radiography centered on the PIPJ of the affected digit as well as AP, lateral, and oblique views of the hand to rule out other injuries. Subtle findings such as the "V" sign, ${ }^{13}$ which is created by the divergence of the dorsal articular surfaces and indicates the presence of dorsal instability, signify that the PIPJ is not concentrically reduced (Figure 4, A). If a depressed articular fracture is suspected but inadequately visualized, radiography under traction or CT may be necessary.

\section{Physical Examination}

Examination of the injured hand includes inspection of all digits for skin compromise, deformity, swelling, ecchymosis, malrotation, or angulation. Puckering of the skin about the joint suggests that soft-tissue structures may be interposed. ${ }^{1}$ Physical examination should focus on checking for localized tenderness to palpation, examining the competency of the soft-tissue structures surrounding the PIPJ and the integrity of flexor and extensor tendons, and determining the neurovascular status of the injured digit. Close attention should be paid to sensation on each side of the injured digit because traction neurapraxia on the stretched side is common. A digital block with local anesthetic may be required to facilitate adequate assessment of the injured joint.

After review of appropriate imaging studies, gentle reduction of the dislocated joint can be attempted. Once reduced, the joint is examined for passive and active stability. The integrity of the VP and CLs should be tested. A radial-directed and then an ulnar-directed force is applied to the middle phalanx to evaluate the integrity of the CLs. Attempted dorsal 
translation of the middle phalanx (ie, PIPJ hyperextension) tests the competency of the VP. ${ }^{4}$ An Elson test is performed to assess the integrity of the central slip; the PIPJ is flexed as far as is comfortably possible (ideally close to $90^{\circ}$ ), and the examiner holds the joint in this position while the patient attempts to extend the distal interphalangeal (DIP) joint against resistance. ${ }^{14}$ Fixed extension of the DIP joint and inability to extend the PIPJ indicates loss of central slip integrity.

Active stability is assessed by asking the patient to actively flex and extend the PIPJ. Completion of near full arc of motion without hinging is an indication of adequate joint stability. Hinging can appear as normal motion and can be detected only on radiographs (Figure 7). At our institution, we obtain standard flexion and extension radiographs of the affected digit when hinging is suspected. MCP and DIP joint motion should also be evaluated because stiffness in these joints may result from the original trauma or as a sequela of management of the PIPJ injury. ${ }^{9,15}$

\section{Management Goals}

Goals of management of fracture-dislocation of the PIPJ include obtaining and maintaining a reduced joint, reestablishing normal joint motion, allowing early range of motion (ROM) if possible without compromising stability and, when feasible, achieving an anatomic reduction of the articular surface. A well-aligned, reduced joint is essential for acceptable clinical outcome. ${ }^{9,12}$

With regard to the articular surface, quality of reduction has not been shown to correlate with clinically relevant posttraumatic arthrosis in the PIPJ. Several investigators have shown that a small intra-articular step-off can be well tolerated ${ }^{16,17}$ in the setting of a well-aligned, reduced joint with no hinging and early initiation of ROM to promote cartilage healing. ${ }^{18}$ The PIPJ is prone to stiffness, and periods of immobilization as short as 4 weeks may result in permanent loss of motion. ${ }^{2}$

Initial evaluation and management of most PIPJ injuries occurs in the field of play, emergency department, or ambulatory setting. Irreducible dislocations should be managed surgically in a timely manner; open injuries require irrigation and débridement.

\section{Nonsurgical Management}

Stable PIPJ fracture-dislocations can be managed nonsurgically. Stable dorsal fracturedislocations can be buddy taped for several weeks, allowing early ROM. ${ }^{19,20}$ Buddy taping of these injuries has been shown to be superior to static immobilization. ${ }^{20}$ In patients with stable volar fracture-dislocations, the extensor mechanism's ability to heal must be considered. The amount of fracture displacement in which extensor mechanism function can be predictably restored via nonsurgical management has not been well defined in the literature. Kang and Stern ${ }^{9}$ suggest that predictable restoration of central slip function can be achieved without surgery in fractures with an avulsed fragment displaced $\_\mathrm{mm}$. The authors managed these injuries by placing a splint dorsally, which immobilized the PIPJ in full extension while allowing motion at the DIP and MCP joints. The PIPJ remained immobilized for 3 to 4 weeks, after which a dynamic extension splint was used for another 2 
weeks, allowing active flexion. ${ }^{9}$ Surgery should be considered when the fracture displacement is large enough to raise concern regarding excessive extensor lag of the PIPJ.

Tenuous dorsal fracture-dislocations with no hinging during ROM are often managed with extension block splinting ${ }^{19}$ (Figure 8 ). The splint prevents extension of the PIPJ into the range where it is unstable while permitting motion within the stable range. No studies have defined a strict criterion for which nonstable PIPJ fracture-dislocations can be reliably managed nonsurgically. Moreover, no high-quality evidence exists in the literature to support the consideration that injuries requiring $>30^{\circ}$ of flexion to maintain stability should undergo surgical stabilization, while those that require $<30^{\circ}$ of flexion should be managed nonsurgically. Acceptable outcomes have been achieved with short periods of immobilization with the finger in as much as $50^{\circ}$ to $60^{\circ}$ of flexion. ${ }^{19}$ Immobilizing the finger in increasing flexion carries the risk of PIPJ flexion contracture; therefore, this risk must be weighed against the risks of surgery. ${ }^{21,22}$ In general, when $>30^{\circ}$ of flexion is required to maintain a stable reduction, surgical intervention should be considered to optimize functional outcome. ${ }^{1,7,9}$

Close follow-up with frequent radiographic examination is warranted to ensure that reduction is maintained. Our preference is to leave the splint in place for 2 weeks and then decrease flexion by $10^{\circ}$ each week. If subluxation occurs, the joint is resplinted in its most recent stable position. Once the joint is stable in full extension, the splint is discontinued and the joint is protected with buddy taping for an additional 3 weeks.

\section{Surgical Management}

\section{Dorsal Fracture-dislocations}

Surgical management is required for dorsal fracture-dislocations in which reduction cannot be maintained (eg, dorsal subluxation, hinging at fracture site). Options include closed reduction and transarticular or extension block pinning, closed reduction and percutaneous pinning (CRPP) of the fracture fragments, dynamic distraction and external fixation (DDEF), open reduction and internal fixation (ORIF), volar plate arthroplasty (VPA), and hemihamate reconstruction arthroplasty (HHRA).

Closed Reduction and Transarticular or Extension Block Pinning-When a PIPJ fracture-dislocation is amendable to closed reduction but concern exists regarding maintenance of reduction via closed means, transarticular or extension block pinning of the PIPJ may be a good option. Overall alignment of the PIPJ is maintained, with little effort made to reduce individual fragments.

Extension block pinning involves inserting a Kirschner wire (K-wire) longitudinally into the distal aspect of the proximal phalanx such that the wire blocks the amount of PIPJ extension required to maintain reduction. ${ }^{23}$ In theory, this technique should allow some motion, but in practice, early motion is difficult to attain. With transarticular pinning, alignment of the injured joint is maintained by placing a K-wire across the PIPJ. 
Closed reduction and pinning trades early motion for stability. Newington et $\mathrm{al}^{24}$ treated 11 unstable dorsal fracture-dislocations with closed reduction and transarticular pinning. In their protocol, a K-wire was used to hold the PIPJ in $20^{\circ}$ to $40^{\circ}$ of flexion for 3 weeks. The wire was then removed, and an extension block splint was used for an additional 2 weeks. At an average follow-up of 16 years, these patients had a mean flexion contracture of $8^{\circ}$ at the PIPJ, with a mean arc of motion of $85^{\circ}$.

Closed Reduction and Percutaneous Pinning-When the fracture consists of one or more large fragments and satisfactory reduction can be obtained with closed means, CRPP of the fracture fragments with transarticular or extension block pinning is an option. Following reduction, the volar fragment is pinned to the middle phalanx with K-wires in either a volar-to-dorsal or dorsal-to-volar direction. PIPJ alignment is maintained with transarticular or extension block pinning.

In a series of six patients with unstable dorsal fracture-dislocations treated with CRPP and extension block pinning, Vitale et $\mathrm{al}^{25}$ reported that reduction was maintained in all patients with no complications reported. The mean arc of motion was from $4^{\circ}$ of extension to $93^{\circ}$ of flexion, and the mean Disabilities of the Arm, Shoulder, and Hand (DASH) score was 8 on scale of zero to 100 , with zero representing no disability.

As with transarticular and extension block pinning, CRPP of individual fracture fragments avoids the soft-tissue trauma associated with open techniques, which may exacerbate joint stiffness. Although it may seem intuitive that a more congruent joint is desirable, reducing and per cutaneously stabilizing individual fracture fragments has not been shown to be superior to simple transarticular or extension block pinning.

Dynamic Distraction and External Fixation-DDEF can be used to manage fracturedislocation of the PIPJ if traction and ligamentotaxis can secure adequate fracture alignment and maintain a well-aligned reduction. Several DDEF designs for the PIPJ have been described in the literature. ${ }^{16,26,27}$ A discussion of the individual merits of these various designs is beyond the scope of this article, but they all share some fundamental properties.

DDEF provides distraction across the joint, employing the principles of ligamentotaxis to align fracture fragments. Distraction off-loads the articular surface. DDEF permits early active and passive ROM, combating stiffness and promoting cartilage healing. As with CRPP, placement of a DDEF device causes minimal soft-tissue trauma. We prefer the DDEF device described by Slade et al. ${ }^{27}$ It is built in the operating room using long $\mathrm{K}$-wires and dental rubber bands and can be removed in the office (Figure 9).

Ellis et al ${ }^{28}$ used the DDEF device developed by Slade et al ${ }^{27}$ to treat eight patients with unstable dorsal fracture-dislocations. The patients regained a mean ROM of $89^{\circ}$ and grip strength was $92 \%$ of the unaffected hand. Ruland et $\mathrm{al}^{29}$ also used the Slade DDEF design to treat 34 active duty members of the US Armed Forces who sustained PIPJ fracturedislocations. The authors reported a mean final arc of motion of $88^{\circ}$, and all patients returned to prior levels of activity and duty. The DDEF can be used alone or to augment 
other forms of surgical management such as ORIF, VPA, or HHRA, ensuring that the fixation or reconstruction is stable enough to allow early ROM. ${ }^{12}$

Open Reduction and Internal Fixation-ORIF with a plate and screws or screws alone, is most likely to be successful in patients with large fracture fragments and little or no comminution. ${ }^{9,30}$ In some cases, fracture fragment size may be smaller than predicted based on preoperative imaging; this may result in tenuous fracture fixation, prolonged immobilization, or an intraoperative change in treatment plan. Impaction-shear type injuries may require bone graft to fill the metaphyseal void created by elevation of the articular surface.

Excellent outcomes have been reported with ORIF of PIPJ fracture-dislocations. ${ }^{31}$ However, it is a technically demanding procedure, and several authors have reported serious complications. ${ }^{8,32}$ In a study of nine patients with dorsal fracture-dislocations treated with ORIF, Hamilton et $\mathrm{al}^{30}$ reported that the mean ROM of the PIPJ was $70^{\circ}$ at final follow-up, with seven of the nine patients completely pain free. Average grip strength was $98 \%$ of that on the contralateral side. In a recent study of 13 patients with unstable dorsal fracture dislocations treated with ORIF, Cheah et al ${ }^{33}$ reported that the mean ROM was $75^{\circ}$, grip strength was $85 \%$ of that on the contralateral side, and the DASH score was 4 at an average 2-year follow-up. However, almost one third of patients required hardware removal.

Volar Plate Arthroplasty_VPA is performed through a volar exposure of the PIPJ. The VP is identified, mobilized from the CLs, and advanced into the volar defect using a pullout wire or suture. Holes are drilled at the lateral margins of the middle phalanx, and sutures are passed from volar to dorsal. These sutures can then be tied over a button on the dorsal middle phalanx, securing the volar plate into the defect. The reconstruction is then protected using either a transarticular K-wire or a dorsal blocking K-wire.

In 1980, Eaton and Malerich ${ }^{34}$ published the outcomes of 24 patients with fracturedislocations of the PIPJ treated with VPA; the authors used the VP to recreate the volar buttress of the middle phalangeal base (Figure 10). The authors reported that patients who underwent surgery within 6 weeks following injury had a mean final ROM of $95^{\circ}$, whereas patients treated $>6$ weeks following injury had a final mean ROM of $78^{\circ}$.

In a later study of long-term outcomes in 17 patients with unstable dorsal PIPJ fracturedislocations treated with VPA, Dionysian and Eaton ${ }^{35}$ reported better results in patients who had surgery within 4 weeks of the injury than in those who underwent surgery $>4$ weeks after injury.

Transarticular K-wire has been used for postoperative protection of the reconstruction; however, this prohibits early motion. Therefore, several authors have advocated using DDEF to protect the reconstruction in the postoperative period..$^{7,9,12}$ Redislocation or subluxation, remains a concern following VPA for fracture-dislocations of the PIPJ, especially when $>50 \%$ of the articular surface is involved. ${ }^{36}$ 
Hemihamate Reconstruction Arthroplasty-First introduced by Hastings et al, ${ }^{37}$ HHRA is the most recently developed technique for reconstructing the volar buttress of the middle phalanx (Figure 11). HHRA makes use of the similarity in articular surface contour of the volar base of the middle phalanx and the dorsal hamate at the point where it articulates with the bases of the fourth and fifth metacarpals. Autograft is harvested from the dorsal distal hamate between the fourth and fifth metacarpal articulation. The width and depth of the autograft is determined by the width and depth of the middle phalangeal defect. Two or three compression screws are used to secure the autograft to the base of the middle phalanx. Postoperatively, a dorsal splint is applied to hold the PIPJ in $20^{\circ}$ of flexion for 2 weeks.

Williams et a ${ }^{38}$ reported on 12 consecutive patients with unstable dorsal fracturedislocations treated with HHRA. Two patients had recurrent dorsal subluxation. Average ROM at the PIPJ was $85^{\circ}$, and 11 of 12 patients were very satisfied with the outcome. Calfee et $\mathrm{al}^{39}$ performed HHRA in 33 patients with a mix of acute and chronic dorsal fracturedislocations. At a 4.5-year follow-up, mean PIPJ motion was $70^{\circ}$, with a mean DASH score of five. Notably, only patients with extensive comminution or pilon injuries had poor outcomes. In a study of eight patients with fracture-dislocations of the PIPJ treated with HHRA, Afendras et $\mathrm{al}^{40}$ reported a mean arc of motion of $67^{\circ}$ at a mean 4-year follow-up. Two patients had severe arthritis based on radiographic criteria, with one having debilitating pain. The remaining seven patients in the study were satisfied with their outcomes.

The immediate stability of a HHRA both at the donor and recipient sites have been verified in a biomechanical study. ${ }^{41}$ However, long-term outcomes are not available. Studies using animal models and similar nonvascularized bone grafts have raised concerns regarding longterm durability of these grafts. ${ }^{42,43}$

\section{Volar Fracture-dislocations}

Unstable volar fracture-dislocations, those with hinging, or those with considerable fracture displacement (ie, central slip disruption) require surgical intervention. Options include transarticular pinning, CRPP, DDEF, or ORIF.

Volar fracture-dislocations with dorsal comminution present treatment challenges. If the concavity of the middle phalangeal base is restored following closed reduction, a transarticular K-wire can be used to immobilize the joint in extension. ${ }^{9}$ Attempts to restore the middle phalangeal geometry via fragment elevation and bone grafting or by tethering the central slip to the defect have been described, but reportedly result in joint stiffness. ${ }^{9,11}$

Stable volar fracture-dislocations with considerable fracture displacement (eg, $>2 \mathrm{~mm}$ as suggested by Kang and Stern ${ }^{9}$ ) or unstable injuries with large fracture fragments and minimal comminution can be managed with CRPP or ORIF., ${ }^{9,44}$ Ideally, fixation should be stable enough to allow early postoperative ROM. Tekkis et $\mathrm{al}^{45}$ reported good outcomes in two patients with volar fracture-dislocations of the PIPJ treated with ORIF and early motion. Average PIPJ motion at final follow-up was from $9^{\circ}$ to $104^{\circ}$ of flexion and grip strength averaged $81 \%$ of that on the contralateral side. 
Unstable injuries with minimal fracture displacement (the fragment moves with the middle phalanx during motion) can be treated with traction via DDEF and immediate ROM. ${ }^{17}$ Movement of the fracture fragment with motion of middle phalanx indicates that the fragment is sufficiently attached to the middle phalangeal base to allow healing even with motion.

\section{Pilon Injuries}

Pilon injuries of the PIPJ are almost always unstable and require surgical intervention. Nondisplaced pilon injuries can be stable and can be managed nonsurgically; however, this type of injury is rare.

The concavity of the middle phalangeal base can often be restored with traction using DDEF. Literature comparing outcomes associated with DDEF versus ORIF is limited. Stern et $\mathrm{al}^{8}$ showed that the functional result in patients treated with DDEF was equivalent to that of those treated with ORIF. The authors found that anatomic reduction of the articular surface is often not feasible with DDEF or ORIF. ${ }^{8}$ Sarris et al ${ }^{46}$ treated four patients with PIPJ pilon fractures using a combination of traction and limited ORIF, consisting of a limited incision with supplemental K-wires used to improve the position of severely displaced fragments where needed or for the repair of the central tendon. At 29-month follow-up, mean ROM was $94^{\circ}$, and only one patient had pain with activities of daily living. Nevertheless, because of the propensity for complications, the use of ORIF for management of volar fracture-dislocations should be considered carefully. ${ }^{8}$

\section{Chronic Injuries}

Chronic PIPJ fracture-dislocations, defined as injuries $>6$ weeks old, present a treatment challenge. ${ }^{34,35,44,47}$ Expected outcome for chronic injuries, regardless of treatment, is worse than that for comparable injuries treated acutely. Several studies of surgical management of PIPJ fracture-dislocations have compared outcomes of acute and chronic injuries and found that chronic injuries fare worse universally.

In a study of ORIF for management of volar lip fracture, Grant et al ${ }^{47}$ compared seven patients treated acutely (within 14 days) with seven patients in which treatment was delayed for an average of 47 days. At final follow-up, the mean ROM was $14^{\circ}$ worse for the delayed group than the acute group $\left(86^{\circ}\right.$ versus $100^{\circ}$, respectively). There was also a $43 \%$ rate of recurrent instability in the delayed group. Rosenstadt et al ${ }^{44}$ treated 13 unstable volar fracture-dislocations, nine were acute injuries and four were chronic. Seven of nine acute injuries were treated with CRPP, resulting in a mean ROM of $91^{\circ}$ with no functional limitations. All of the chronic injuries necessitated ORIF. The authors reported a mean ROM of $70^{\circ}$, with 2 of 4 patients exhibiting flexion contractures.

Among patients who underwent VPA in the study by Eaton and Malerich, ${ }^{34}$ those treated within 6 weeks of injury had a better outcome than those treated 6 weeks after injury. Similarly, Dionysian and Eaton ${ }^{35}$ reported better outcomes in patients with acute injuries than in those with chronic injuries. 


\section{Rehabilitation}

Rehabilitation is a vital component of management of PIPJ fracture-dislocations. Schenck et $\mathrm{al}^{48}$ found that, in patients with pilon injuries treated with traction and early passive movement exercises, mean ROM improved from $50^{\circ}$ upon discontinuation of traction to $87^{\circ}$ at final follow-up.

Although rehabilitation protocol varies based on the type of injury and treatment modality, some generalizations can be made. With the notable exception of injuries to the central slip and management methods employing a transarticular K-wire, active ROM within the stable arc of motion should be initiated as early as allowed by the stability of the fixation construct. Some authors have reported successful outcomes with up to 4 weeks of immobilization. ${ }^{23,25}$ Others believe that static immobilization for more than 3 weeks can result in permanent loss of motion. ${ }^{2}$ Active-assist and passive ROM exercises are not typically used in the immediate postoperative period because they place stress on healing fractures and soft tissues. In injuries treated with closed reduction and extension block splinting, active ROM within the stable range is allowed immediately. Similarly, immediate active ROM is allowed in fracture-dislocations managed with DDEF.

\section{Summary}

The goal of early diagnosis and timely management of fracture-dislocations of the PIPJ is to establish a well-aligned, reduced joint. Chronic injuries present treatment challenges and outcomes are often poor. The orthopaedic surgeon has a large armamentarium of nonsurgical and surgical techniques to choose from, allowing treatment to be tailored to the individual patient and injury. Procedures such as VPA and, in particular, HHRA can be used both as the primary treatment modality and as salvage procedures following failure of another treatment modality. Some loss of motion is expected in nearly all cases of PIPJ fracture-dislocations, regardless of treatment. Nevertheless, a functional, pain-free PIPJ is a reasonable treatment goal in patients with acute injuries.

\section{Acknowledgments}

Dr. Elfar or an immediate family member has received research or institutional support from Synthes and Arthrex, and serves as a board member, owner, officer, or committee member of the American Society for Surgery of the Hand and the J. Robert Gladden Orthopaedic Society. Neither Dr. Mann nor any immediate family member has received anything of value from or has stock or stock options held in a commercial company or institution related directly or indirectly to the subject of this article.

\section{References}

Evidence-based Medicine: Levels of evidence are described in the table of contents. In this article, references 12 and 32 are level II studies. References 11, 34, 36, and 44 are level III studies. References 14, 16, 17, 19, 20, 22-26, 28-31, 33, 35, 38-41, and 45-48 are level IV studies.

References printed in bold type are those published within the past 5 years.

1. Green, DP. Green's Operative Hand Surgery. 6. Philadelphia, PA: Churchill Livingstone; 2005. 
2. Strickland, JW., Steichen, JB. American Society for Surgery of the Hand. Difficult Problems in Hand Surgery. St. Louis, MO: Mosby; 1982. p. xiiip. 434

3. Leibovic SJ, Bowers WH. Anatomy of the proximal interphalangeal joint. Hand Clin. 1994; 10(2): 169-178. [PubMed: 8040195]

4. Bowers WH, Wolf JW Jr, Nehil JL, Bittinger S. The proximal interphalangeal joint volar plate: I. An anatomical and biomechanical study. J Hand Surg Am. 1980; 5(1):79-88. [PubMed: 7365222]

5. Kiefhaber TR, Stern PJ, Grood ES. Lateral stability of the proximal interphalangeal joint. J Hand Surg Am. 1986; 11(5):661-669. [PubMed: 3760491]

6. Trumble, T. Principles of Hand Surgery and Therapy. Philadelphia, PA: WB Saunders; 2000. p. xvip. 644

7. Kiefhaber, TR. Phalangeal Dislocations/Periarticular Trauma. New York, NY: McGraw-Hill Professional Publishing; 1996.

8. Stern PJ, Roman RJ, Kiefhaber TR, McDonough JJ. Pilon fractures of the proximal interphalangeal joint. J Hand Surg Am. 1991; 16(5):844-850. [PubMed: 1940163]

9. Kang R, Stern PJ. Fracture dislocations of the proximal interphalangeal joint. Journal of the American Society for Surgery of the Hand. 2002; 2:47-59.

10. Akagi T, Hashizume H, Inoue H, Ogura T, Nagayama N. Computer simulation analysis of fracture dislocation of the proximal interphalangeal joint using the finite element method. Acta Med Okayama. 1994; 48(5):263-270. [PubMed: 7863798]

11. Hastings H II, Carroll C IV. Treatment of closed articular fractures of the metacarpophalangeal and proximal interphalangeal joints. Hand Clin. 1988; 4(3):503-527. [PubMed: 3170661]

12. Krakauer JD, Stern PJ. Hinged device for fractures involving the proximal interphalangeal joint. Clin Orthop Relat Res. 1996; (327):29-37.

13. Light TR. Buttress pinning techniques. Orthopaedic Review. 1981; 10:49-55.

14. Elson RA. Rupture of the central slip of the extensor hood of the finger: A test for early diagnosis. J Bone Joint Surg Br. 1986; 68(2):229-231. [PubMed: 3958008]

15. Calfee RP, Sommerkamp TG. Fracture-dislocation about the finger joints. J Hand Surg Am. 2009; 34(6):1140-1147. [PubMed: 19643295]

16. Agee JM. Unstable fracture dislocations of the proximal interphalangeal joint: Treatment with the force couple splint. Clin Orthop Relat Res. 1987; (214):101-112. [PubMed: 3791731]

17. Morgan JP, Gordon DA, Klug MS, Perry PE, Barre PS. Dynamic digital traction for unstable comminuted intra-articular fracture-dislocations of the proximal interphalangeal joint. J Hand Surg Am. 1995; 20(4):565-573. [PubMed: 7594279]

18. Salter RB. The physiologic basis of continuous passive motion for articular cartilage healing and regeneration. Hand Clin. 1994; 10(2):211-219. [PubMed: 8040199]

19. McElfresh EC, Dobyns JH, O'Brien ET. Management of fracture-dislocation of the proximal interphalangeal joints by extension-block splinting. J Bone Joint Surg Am. 1972; 54(8):17051711. [PubMed: 4653646]

20. Phair IC, Quinton DN, Allen MJ. The conservative management of volar avulsion fractures of the P.I.P. joint. J Hand Surg Br. 1989; 14(2):168-170. [PubMed: 2746116]

21. Kuczynski K. The proximal interphalangeal joint: Anatomy and causes of stiffness in the fingers. J Bone Joint Surg Br. 1968; 50(3):656-663. [PubMed: 5726917]

22. Peimer CA, Sullivan DJ, Wild DR. Palmar dislocation of the proximal interphalangeal joint. J Hand Surg Am. 1984; 9(1):39-48.

23. Viegas SF. Extension block pinning for proximal interphalangeal joint fracture dislocations: Preliminary report of a new technique. J Hand Surg Am. 1992; 17(5):896-901. [PubMed: 1401802]

24. Newington DP, Davis TR, Barton NJ. The treatment of dorsal fracture-dislocation of the proximal interphalangeal joint by closed reduction and Kirschner wire fixation: A 16-year follow up. J Hand Surg Br. 2001; 26(6):537-540. [PubMed: 11884107]

25. Vitale MA, White NJ, Strauch RJ. A percutaneous technique to treat unstable dorsal fracturedislocations of the proximal interphalangeal joint. J Hand Surg Am. 2011; 36(9):1453-1459. [PubMed: 21820818] 
26. Deshmukh SC, Kumar D, Mathur K, Thomas B. Complex fracture-dislocation of the proximal interphalangeal joint of the hand: Results of a modified pins and rubbers traction system. J Bone Joint Surg Br. 2004; 86(3):406-412. [PubMed: 15125130]

27. Slade JF, Baxamusa TH, Wolf SW. External fixation of proximal interphalangeal joint fracturedislocations. Atlas of the Hand Clinics. 2000; 5:1-29.

28. Ellis SJ, Cheng R, Prokopis P, et al. Treatment of proximal interphalangeal dorsal fracturedislocation injuries with dynamic external fixation: A pins and rubber band system. J Hand Surg Am. 2007; 32(8):1242-1250. [PubMed: 17923310]

29. Ruland RT, Hogan CJ, Cannon DL, Slade JF. Use of dynamic distraction external fixation for unstable fracture-dislocations of the proximal interphalangeal joint. J Hand Surg Am. 2008; 33(1): 19-25. [PubMed: 18261660]

30. Hamilton SC, Stern PJ, Fassler PR, Kiefhaber TR. Mini-screw fixation for the treatment of proximal interphalangeal joint dorsal fracture-dislocations. J Hand Surg Am. 2006; 31(8):13491354. [PubMed: 17027798]

31. Wolfe SW, Katz LD. Intra-articular impaction fractures of the phalanges. J Hand Surg Am. 1995; 20(2):327-333. [PubMed: 7775779]

32. Aladin A, Davis TR. Dorsal fracture-dislocation of the proximal interphalangeal joint: A comparative study of percutaneous Kirschner wire fixation versus open reduction and internal fixation. J Hand Surg Br. 2005; 30(2):120-128. [PubMed: 15757762]

33. Cheah AE, Tan DM, Chong AK, Chew WY. Volar plating for unstable proximal interphalangeal joint dorsal fracture-dislocations. J Hand Surg Am. 2012; 37(1):28-33. [PubMed: 22018477]

34. Eaton RG, Malerich MM. Volar plate arthroplasty of the proximal interphalangeal joint: A review of ten years' experience. J Hand Surg Am. 1980; 5(3):260-268. [PubMed: 7400563]

35. Dionysian E, Eaton RG. The long-term outcome of volar plate arthroplasty of the proximal interphalangeal joint. J Hand Surg Am. 2000; 25(3):429-437. [PubMed: 10811746]

36. Deitch MA, Kiefhaber TR, Comisar BR, Stern PJ. Dorsal fracture dislocations of the proximal interphalangeal joint: Surgical complications and long-term results. J Hand Surg Am. 1999; 24(5): 914-923. [PubMed: 10509268]

37. Hastings, H., Capo, J., Steinberg, B., Stern, P. Hemicondylar hamate replacement arthroplasty for proximal interphalangeal joint fracture/dislocations. Presented at the 54th Annual Meeting of the American Society for Surgery of the Hand; Boston, MA. September 2-4, 1999;

38. Williams RM, Kiefhaber TR, Sommerkamp TG, Stern PJ. Treatment of unstable dorsal proximal interphalangeal fracture/dislocations using a hemi-hamate autograft. J Hand Surg Am. 2003; 28(5):856-865. [PubMed: 14507519]

39. Calfee RP, Kiefhaber TR, Sommerkamp TG, Stern PJ. Hemi-hamate arthroplasty provides functional reconstruction of acute and chronic proximal interphalangeal fracture-dislocations. J Hand Surg Am. 2009; 34(7):1232-1241. [PubMed: 19700071]

40. Afendras G, Abramo A, Mrkonjic A, Geijer M, Kopylov P, Tägil M. Hemi-hamate osteochondral transplantation in proximal interphalangeal dorsal fracture dislocations: A minimum 4 year followup in eight patients. J Hand Surg Eur Vol. 2010; 35(8):627-631. [PubMed: 20511322]

41. Capo JT, Hastings H II, Choung E, Kinchelow T, Rossy W, Steinberg B. Hemicondylar hamate replacement arthroplasty for proximal interphalangeal joint fracture dislocations: An assessment of graft suitability. J Hand Surg Am. 2008; 33(5):733-739. [PubMed: 18590857]

42. Lane JG, Massie JB, Ball ST, et al. Follow-up of osteochondral plug transfers in a goat model: A 6month study. Am J Sports Med. 2004; 32(6):1440-1450. [PubMed: 15310569]

43. Kleemann RU, Schell H, Thompson M, Epari DR, Duda GN, Weiler A. Mechanical behavior of articular cartilage after osteochondral autograft transfer in an ovine model. Am J Sports Med. 2007; 35(4):555-563. [PubMed: 17293465]

44. Rosenstadt BE, Glickel SZ, Lane LB, Kaplan SJ. Palmar fracture dislocation of the proximal interphalangeal joint. J Hand Surg Am. 1998; 23(5):811-820. [PubMed: 9763254]

45. Tekkis PP, Kessaris N, Gavalas M, Mani GV. The role of mini-fragment screw fixation in volar dislocations of the proximal interphalangeal joint. Arch Orthop Trauma Surg. 2001; 121(1-2): 121-122. [PubMed: 11195112] 
46. Sarris I, Goitz RJ, Sotereanos DG. Dynamic traction and minimal internal fixation for thumb and digital pilon fractures. J Hand Surg Am. 2004; 29(1):39-43. [PubMed: 14751101]

47. Grant I, Berger AC, Tham SK. Internal fixation of unstable fracture dislocations of the proximal interphalangeal joint. J Hand Surg Br. 2005; 30(5):492-498. [PubMed: 15990207]

48. Schenck RR. Dynamic traction and early passive movement for fractures of the proximal interphalangeal joint. J Hand Surg Am. 1986; 11(6):850-858. [PubMed: 3794242] 


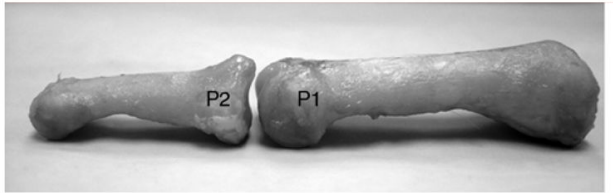

A

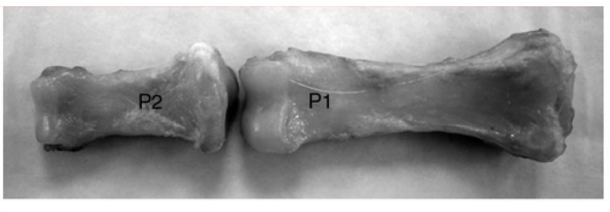

B

Figure 1.

Photographs of the lateral (A) and volar (B) views of the proximal interphalangeal joint (PIPJ). The head of the proximal phalanx (P1) is bicondylar and roughly cylindrical in the sagittal plane. The base of the middle phalanx (P2) is biconcave and has a centrally located intercondylar ridge. The contours of this bony anatomy impart some stability to the PIPJ. (Reproduced from Hogan CH, Nunley JA: Posttraumatic proximal interphalangeal joint flexion contractures. J Am Acad Orthop Surg 2006;14[9]:524-533.) 


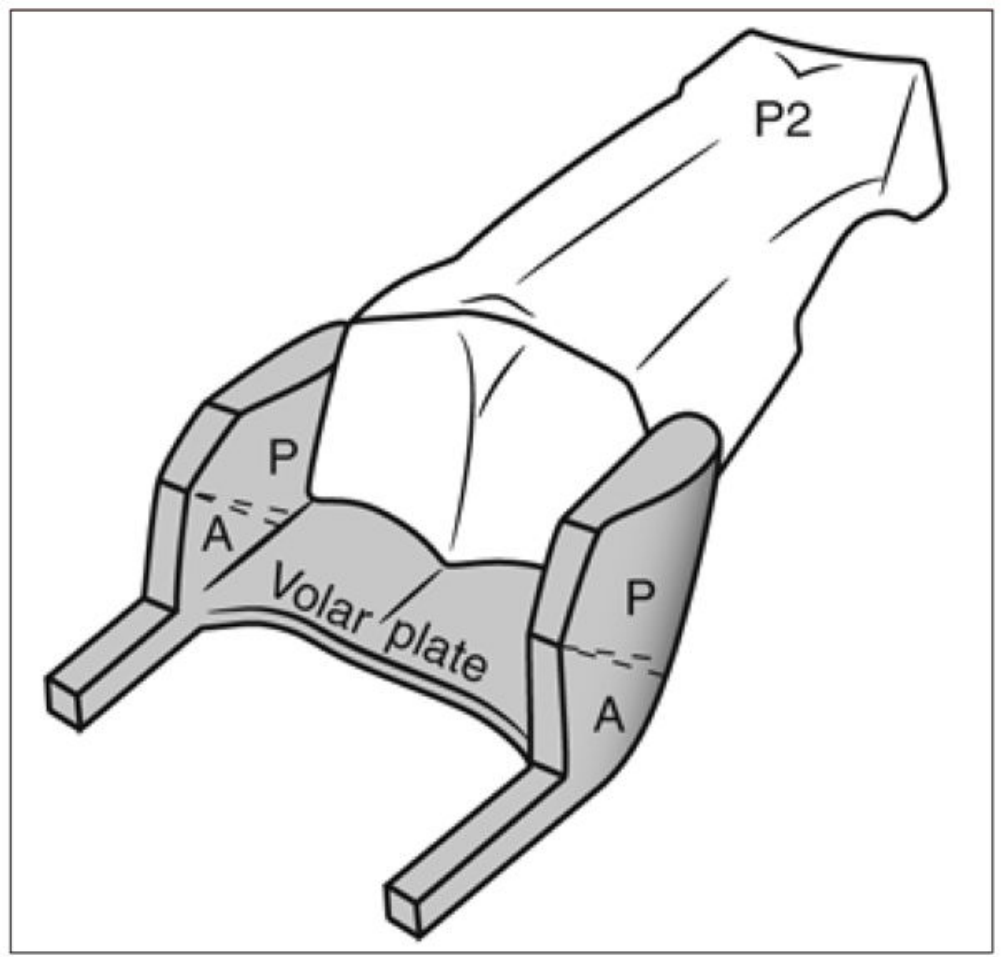

Figure 2.

The collateral ligaments (CLs) and volar plate (VP) form a three-sided box, which is the key to proximal interphalangeal joint (PIPJ) stability. The floor and walls of this box are formed by the VP and CLs, respectively; at least two sides of the box must be injured for instability to occur. The force from the extrinsic and intrinsic tendons crossing the PIPJ also imparts some stability. $\mathrm{A}=$ accessory collateral ligament, $\mathrm{P}=$ proper collateral ligament, $\mathrm{P} 2=$ middle phalanx 


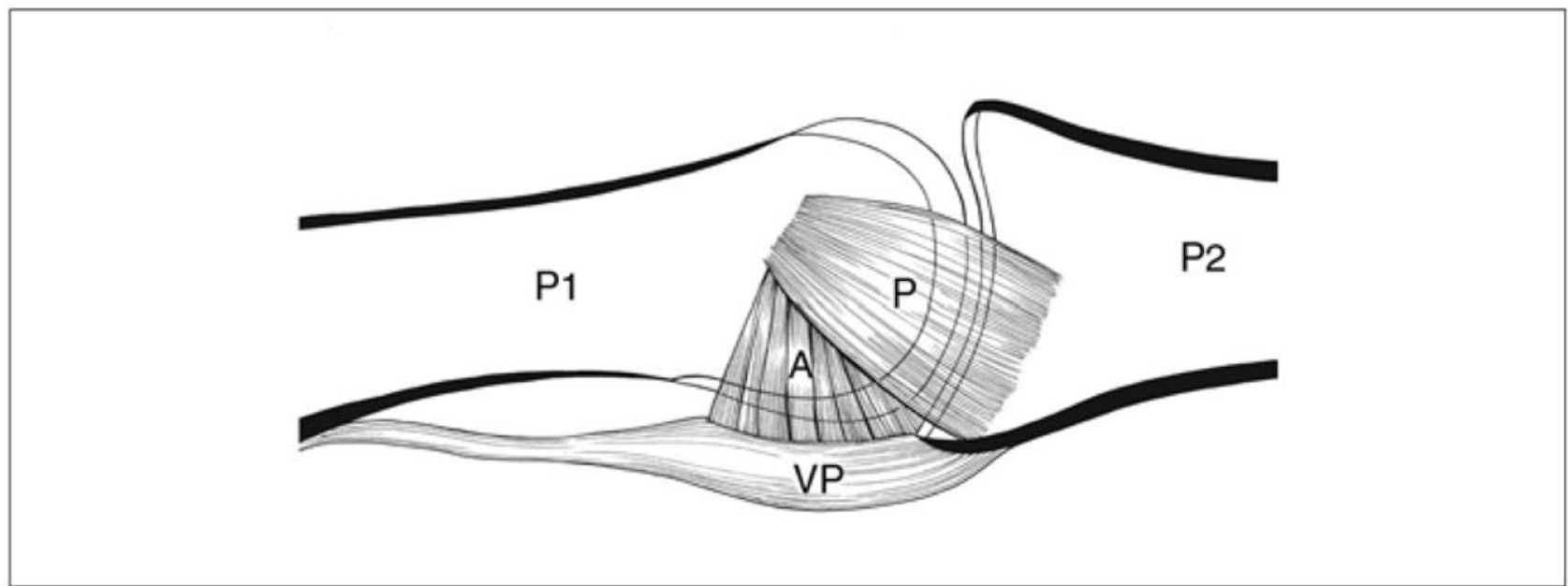

Figure 3.

Illustration of the lateral view of the proximal interphalangeal joint demonstrating the insertion of the proper collateral ligament $(\mathrm{P})$ on the volar third of the middle phalangeal base. The accessory ligament (A) blends with the volar plate (VP). In extension, the accessory ligament is taut and serves as the primary restraint against lateral displacement of the middle phalanx (P2). In flexion, the accessory ligament loosens and, as the proper collateral ligament tightens over the condyles, it assumes the role of primary stabilizer against lateral displacement. P1 = proximal phalanx. (Reproduced from Hogan $\mathrm{CH}$, Nunley JA: Posttraumatic proximal interphalangeal joint flexion contractures. J Am Acad Orthop Surg 2006;14[9]:524-533.) 


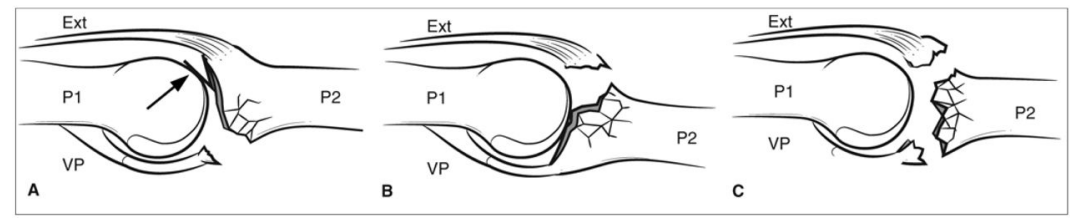

Figure 4.

Illustrations of proximal interphalangeal joint (PIPJ) fracture-dislocation patterns. A, Dorsal PIPJ fracture-dislocation. The middle phalanx (P2) is dislocated or subluxated dorsally, and the volar lip is fractured at its base. The "V" sign (arrow) indicates dorsal instability. Note that the volar plate (VP) attachment is involved in the fracture. B, Volar PIPJ fracturedislocation. The middle phalanx (P2) is dislocated or subluxated volarly and the dorsal lip is fractured. Note the involvement of the central slip attachment (Ext) in the fracture. C, Pilon injury to the PIPJ. The buttressing effects of both the volar and dorsal lips of the middle phalanx are lost. There is comminution of the entire articular surface, and the stabilizing effects of both the VP and central slip are lost, making the injury grossly unstable. P1 = proximal phalanx 


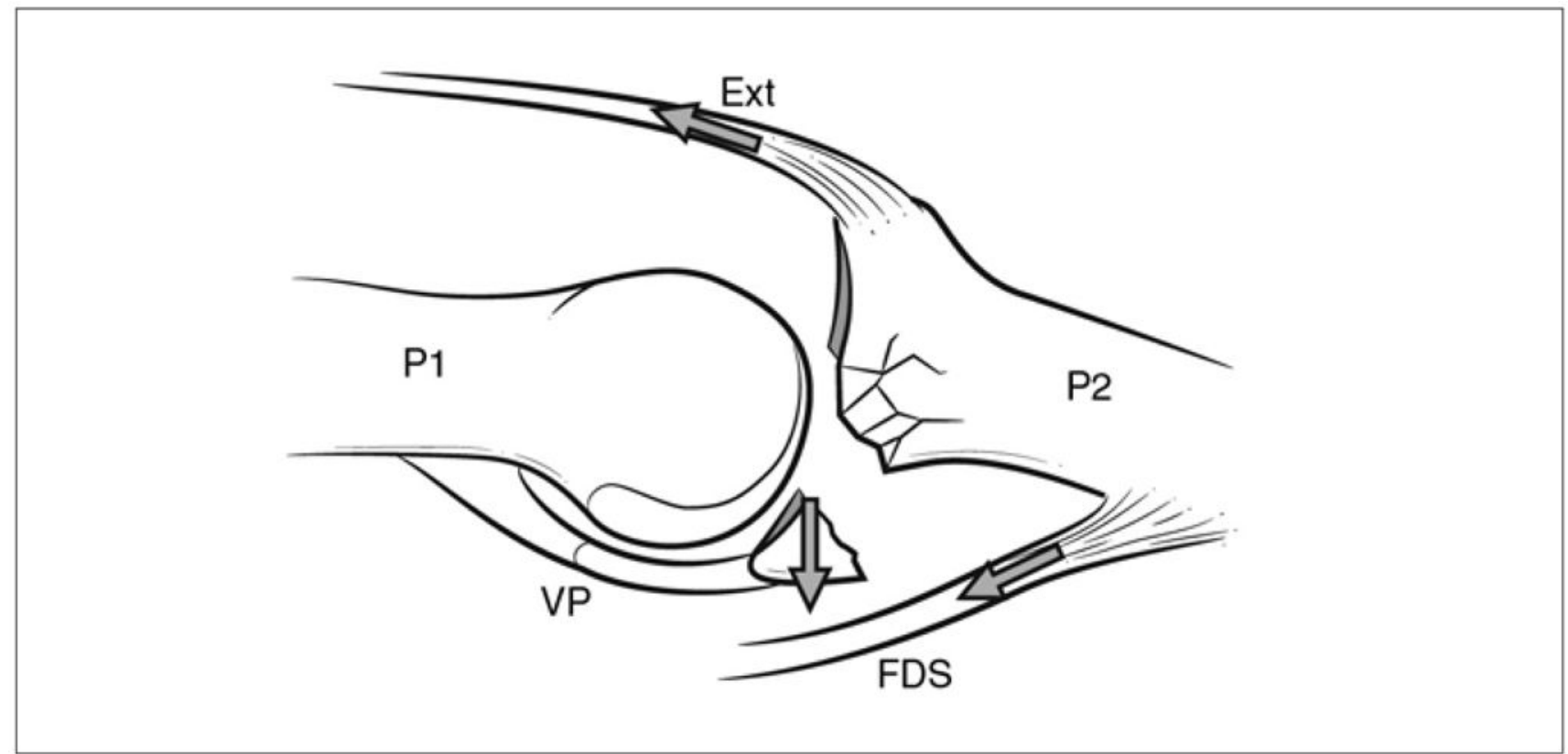

Figure 5.

Illustration demonstrating the forces acting on a dorsal fracture-dislocation of the proximal interphalangeal joint. The tension balance may be altered in the injured joint, resulting in increased instability. The pull of the flexor digitorum superficialis (FDS) tendon flexes the middle phalanx (P2), tilting its base dorsally. This allows the central slip (Ext) to pull the middle phalanx base into a dorsally subluxated position. $\mathrm{P} 1=$ proximal phalanx, $\mathrm{VP}=$ volar plate 


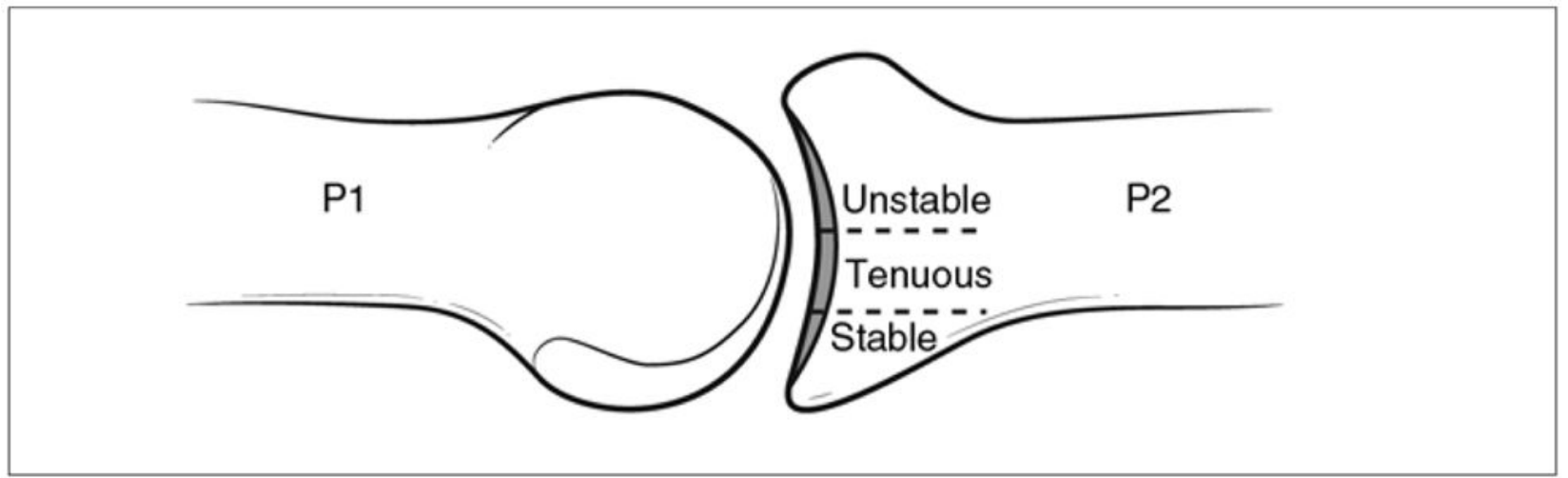

Figure 6.

Illustration demonstrating classification of dorsal fracture-dislocations based on the amount of articular surface involvement. Fractures that involve $<30 \%$ of the articular surface are typically stable whereas those involving $>50 \%$ are often unstable. Stability of fractures that involve $30 \%$ to $50 \%$ of the articular surface is more difficult to predict. These fractures are considered to have tenuous stability. $\mathrm{P} 1=$ proximal phalanx, $\mathrm{P} 2=$ middle phalanx 


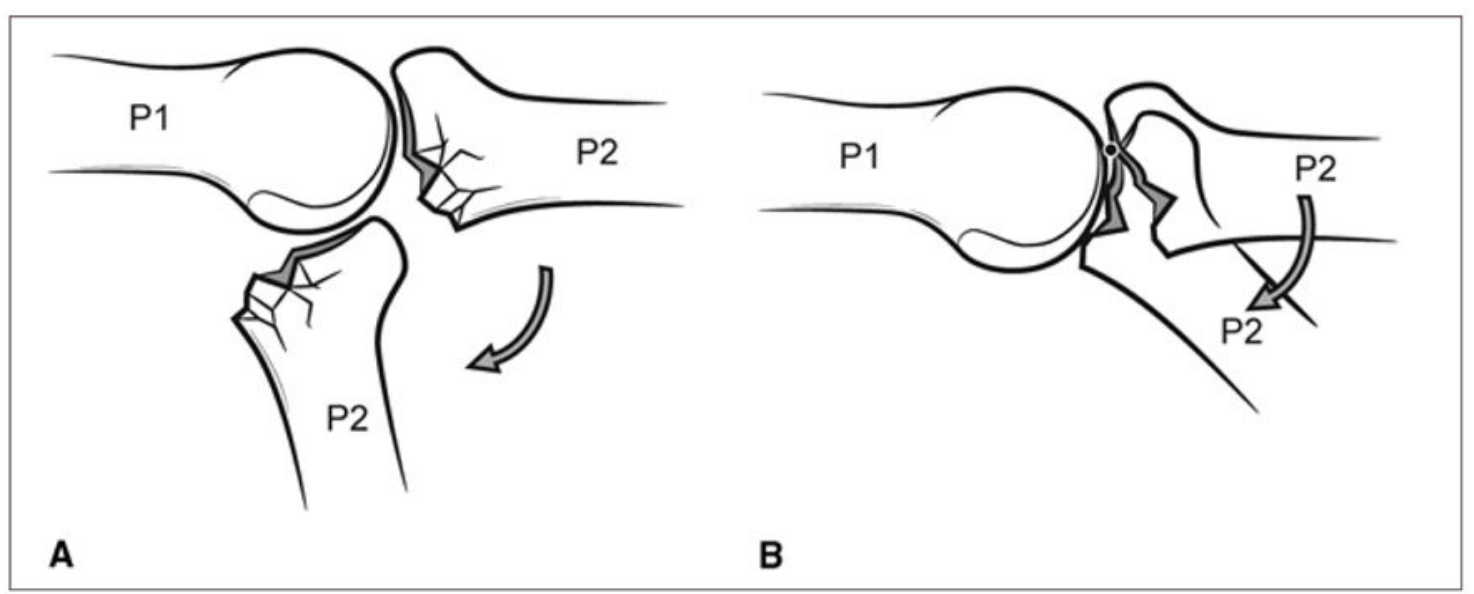

Figure 7.

Illustrations demonstrating proximal interphalangeal joint range of motion without hinging (A) and with hinging (B) following an intra-articular fracture. These fractures may disrupt normal joint motion by causing the middle phalanx (P2) to hinge on the fracture margin (arrow), with normal rotation of the middle phalanx on the proximal side replaced by abnormal translation across the fracture margin. In these cases, joint motion may appear stable, but radiographic examination will reveal hinging. The black dot in panel B is the hinge point. $\mathrm{P} 1$ = proximal phalanx 


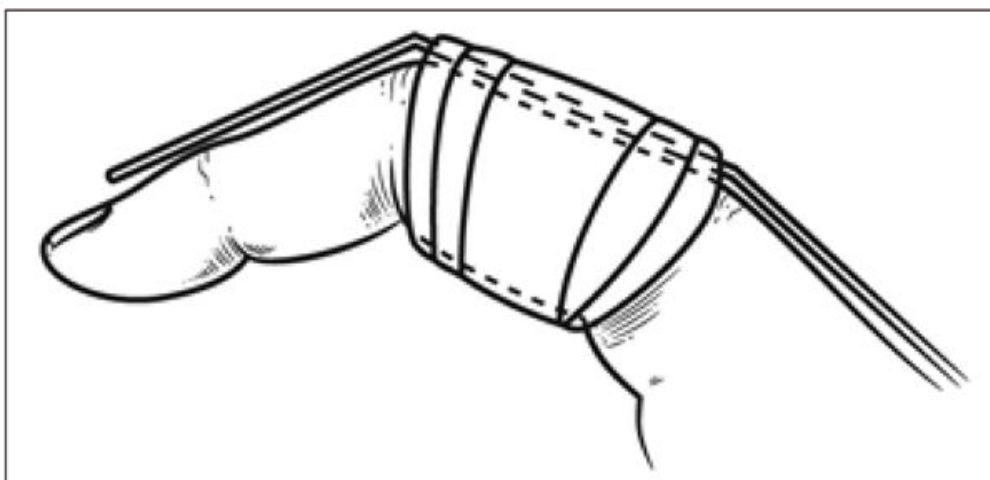

A

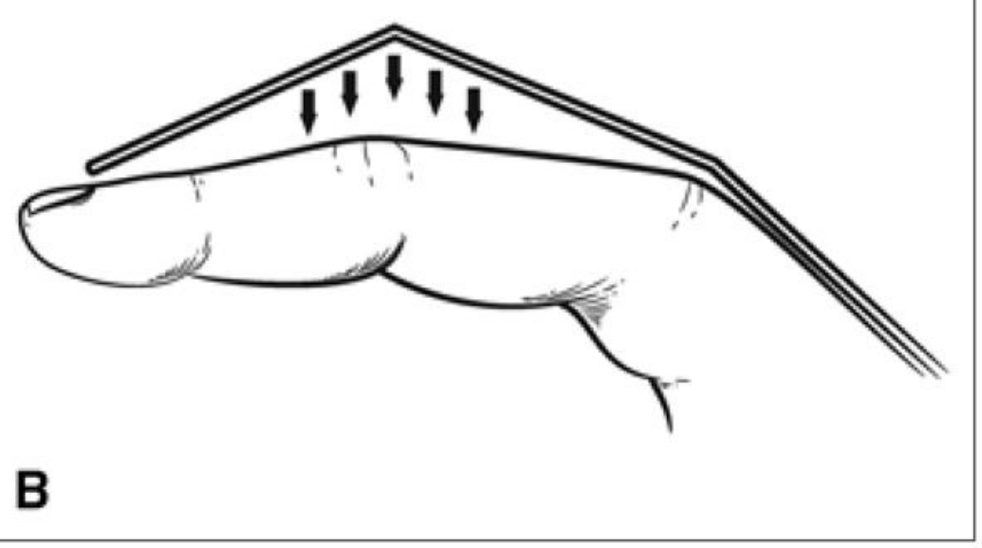

Figure 8.

Illustrations demonstrating correct (A) and incorrect (B) application of an external block splint. The splint must be properly affixed to the proximal phalanx to prevent it from pulling away from the splint and allowing the proximal interphalangeal joint to extend past the intended range, which may result in loss of reduction. 


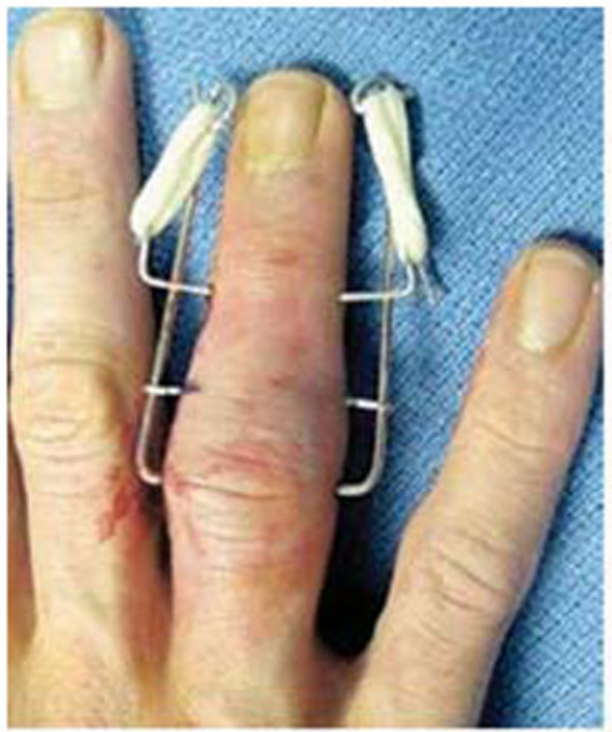

A

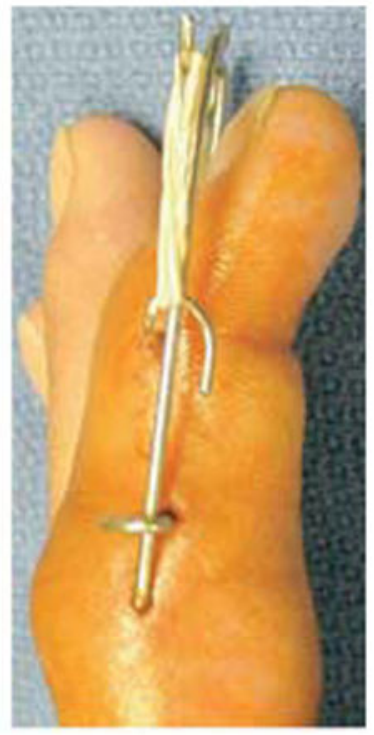

B

\section{Figure 9.}

AP (A) and lateral (B) photographs of a dynamic distraction external fixation device applied to the ring finger. Three Kirschner wires are placed transversely across the bone. The first wire is inserted through the rotational center of the proximal phalangeal head. The second wire is inserted through the center of the middle phalanx distal to the fracture. The final wire is placed through the axis of rotation of the middle phalangeal head. The wires are then bent and dental rubber bands are applied. (Reproduced with permission from Ruland RT, Hogan CJ, Cannon DL, Slade JF: Use of dynamic distraction external fixation for unstable fracturedislocations of the proximal interphalangeal joint. J Hand Surg Am 2008;33[1]:19-25.) 


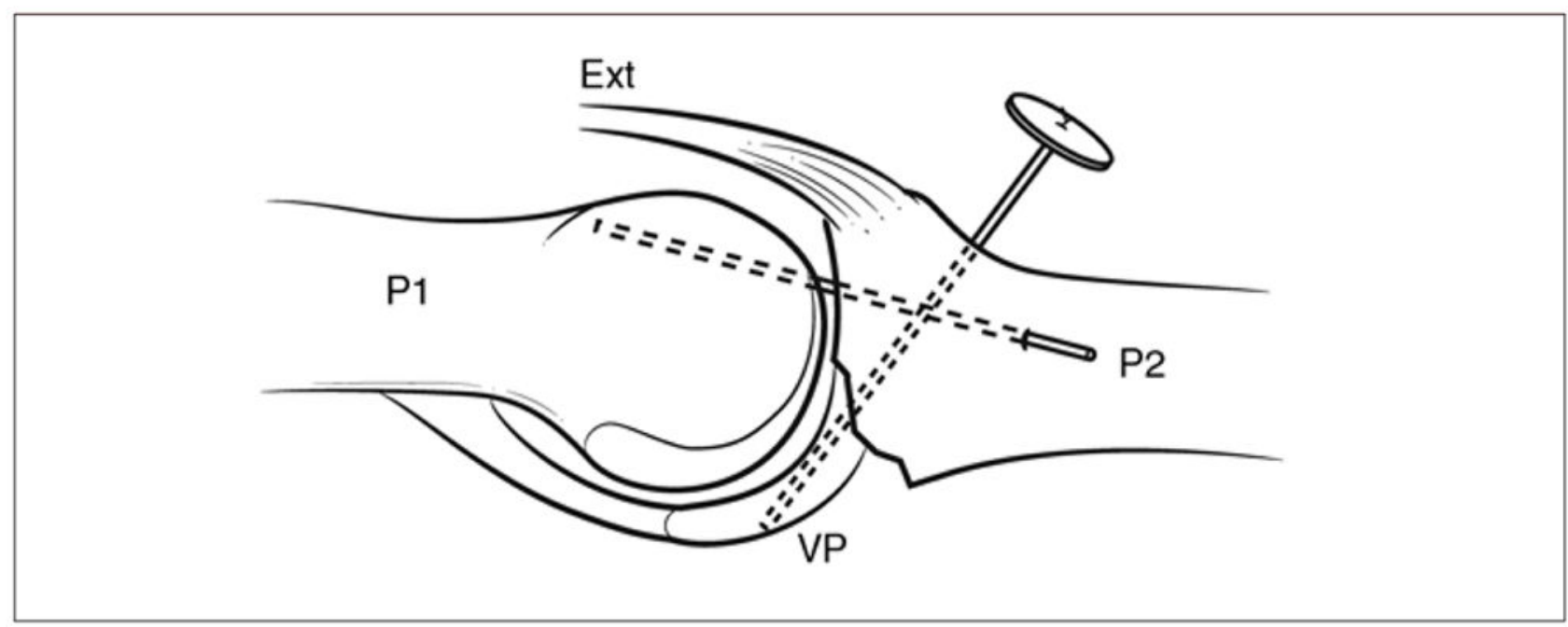

Figure 10.

Illustration demonstrating volar plate arthroplasty for dorsal fracture-dislocation of the proximal interphalangeal joint. A suture is placed through the volar plate (VP) and then attached with a button to the dorsal aspect of the finger. The VP is freed from the collateral ligaments, and a suture is placed through the VP. The traction of the suture will advance the VP. To protect the reconstruction, the joint is immobilized with a transarticular Kirschner wire. Ext $=$ central slip, $\mathrm{P} 1=$ proximal phalanx, $\mathrm{P} 2=$ middle phalanx 

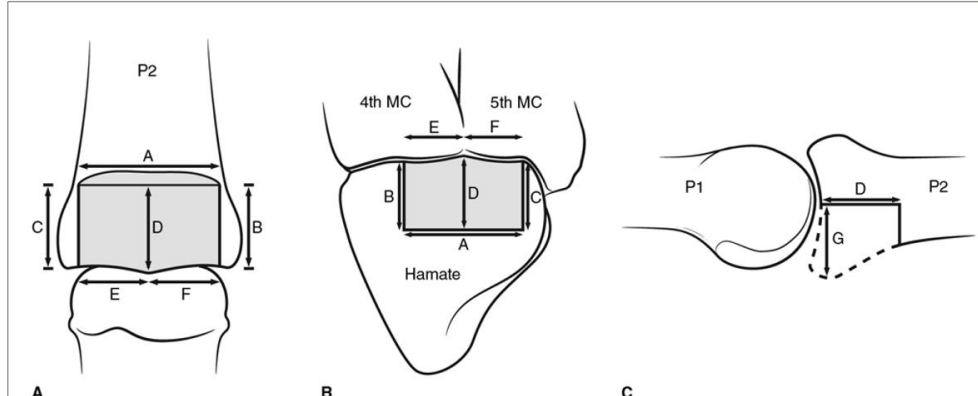

Figure 11.

A, Illustration of the volar view of the proximal interphalangeal joint (PIPJ) demonstrating the dimensions of the defect at the base of the middle phalanx (A through G). Hemihamate reconstruction arthroplasty makes use of the similarity in the articular surface contour between the volar base of the middle phalanx (P2) and the dorsal hamate where it articulates with the bases of the fourth and fifth metacarpals (MC). Illustrations of the dorsal (B) and lateral $(\mathbf{C})$ views of the PIPJ demonstrating the dimensions of the autograft harvested from the dorsal distal hamate. The width and depth of the autograft correspond to that of the defect. $\mathrm{P} 1$ = proximal phalanx 
Table 1

Classification of Proximal Interphalangeal Joint Fracture-dislocations

\begin{tabular}{|c|c|c|c|}
\hline Fracture Type & Clinical Stability & Articular Surface Involvement (\%) & Classification \\
\hline \multirow[t]{3}{*}{ Dorsal } & Stable with full active ROM & $<30$ & Stable \\
\hline & Requires $30^{\circ}$ of flexion to maintain reduction & $30-50$ & Tenuous \\
\hline & Requires $>30^{\circ}$ of flexion to maintain reduction & $>50$ & Unstable \\
\hline \multirow[t]{2}{*}{ Volar } & Stable in full extension & 50 & Stable \\
\hline & Not stable in full extension & $>50$ & Unstable \\
\hline \multirow[t]{2}{*}{ Pilon } & Stable through full active ROM & 100 & Stable \\
\hline & Grossly unstable & 100 & Unstable \\
\hline
\end{tabular}

ROM = range of motion 